\title{
Fiebre, ictericia y hepatitis: no siempre es infección viral
}

\section{Fever, Jaundice and Hepatitis: It is not always a Viral Infection}

\author{
Lina Paola León S., MD, ${ }^{1}$ William Otero, MD, ${ }^{2}$ Martín Gómez, MD. ${ }^{3}$
}

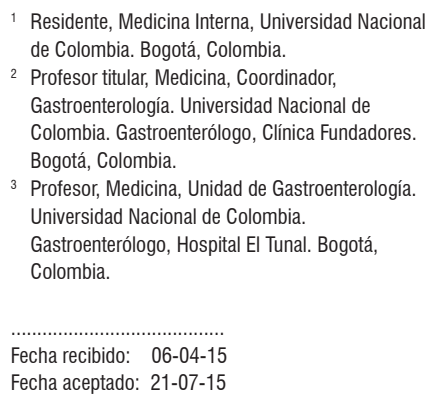

\begin{abstract}
Resumen
La fiebre tifoidea es una infección sistémica causada por Salmonella entérica serotipo tiphy a través de la ingesta de alimentos contaminados (fecal-oral). Es un problema de salud pública, especialmente en países en vías de desarrollo, debido a su alta prevalencia, relacionada con deficiencias sanitarias. En pacientes con fiebre tifoidea, la hepatitis se presenta en $1 \%$ a $26 \%$ de los pacientes y se debe sospechar cuando coexisten ictericia, fiebre y hepatitis. No obstante ser difícil de distinguir de las hepatitis por virus hepatotrópicos, la clave para el diagnóstico es que, en estas infecciones virales, la fiebre desaparece cuando el paciente presenta ictericia. El diagnóstico final de la entidad necesita el aislamiento de la Salmonella a través de coprocultivos y hemocultivos, especialmente de la médula ósea, que es el sitio con mayor rendimiento para el diagnóstico.
\end{abstract}

\section{Palabras clave}

Hepatitis, Salmonella, fiebre entérica.

\begin{abstract}
Typhoid fever is a systemic infection caused by Salmonella enterica serotype Typhi as the result of ingestion of contaminated food (fecal-oral). It is a public health problem, especially in developing countries where it is very prevalent and where it is related to poor sanitation. Hepatitis occurs in $1 \%$ to $26 \%$ of patients with typhoid, so typhoid fever should be suspected when jaundice, fever and hepatitis coexist. Nevertheless, it is difficult to distinguish it from hepatotropic hepatitis virus. The key to diagnosis is that fever disappears in these viral infections while the patient still has jaundice. Final diagnosis requires the isolation of salmonella through blood and/or cultures, but most importantly cultures of bone marrow which have the highest diagnostic yield.
\end{abstract}

\section{Keywords}

Hepatitis, salmonella, enteric fever.

\section{INTRODUCCIÓN}

En pacientes con fiebre tifoidea, el perfil hepático se encuentra alterado en el $21-60 \%$ de ellos (1). Sin embargo, un cuadro de hepatitis aguda solo se encuentra en el 1-26\% de los pacientes (1-3). Cuando hay hepatitis aguda, es más frecuente la recurrencia de la enfermedad $(2,4)$. A pesar de que las manifestaciones clínicas de la hepatitis son indistinguibles de aquellas que son causa- das por virus hepatotropos (A, B, C, D, E) $(2,4,5)$, su diagnóstico puede sospecharse cuando un paciente tiene simultáneamente fiebre, ictericia y hepatitis (aumento de las transaminasas). En las hepatitis producidas por virus hepatropos, cuando aparece la ictericia desaparecen los sintomas generales, incluida la fiebre (6). En esos casos, hay que descartar también otras causas como fiebre amarilla, dengue, varicela o malaria $(2,6)$. Además de la coexistencia de la fiebre con ictericia y hepatitis, son 
claves para el diagnóstico las características de la fiebre tifoidea per se. El diagnóstico definitivo requiere del aislamiento microbiológico por cultivos, siendo el de medula ósea el estándar de oro $(2,4,5)$.

El compromiso hepático por Salmonella fue descrito por primera vez en 1889 por William Osler, cuando reportó 8 casos de hepatomegalia e ictericia en 1500 pacientes con fiebre entérica (2). A este compromiso hepático secundario de la infección por Salmonella se le conoce como hepatitis por Salmonella o hepatitis tifoidea. La hepatitis, en el escenario de infección por Salmonella, es una entidad de buen pronóstico cuando se inicia la antibioticoterapia oportunamente; de lo contrario, la mortalidad puede llegar al $20 \%$ cuando se retrasa el tratamiento en pacientes con manifestaciones severas como perforación intestinal y hemorragia $(1,2,7)$. El compromiso hepático ocurre más frecuentemente en pacientes con inmunosupresión previa, siendo 15 a 100 veces más frecuente en aquellos con VIH $(2,7)$; de ahí la importancia del diagnóstico temprano de esta manifestación extraintestinal en un paciente con fiebre entérica (2).

\section{Epidemiología e impacto}

La fiebre tifoidea es una enfermedad sistémica que se produce con la ingesta de alimentos contaminados por Salmonella typhi o paratiphy (2). Dada su forma de transmisión (fecal-oral), esta patología es reconocida como un importante problema de salud pública en países tropicales y en vías de desarrollo (3) y compromete aproximadamente de 12 a 33 millones de casos en el mundo. En Colombia se reporta una incidencia de 0,22 casos por 100000 habitantes para el 2011, siendo una incidencia baja probablemente relacionada con el subregistro de los casos (8), mientras que en Estados Unidos se reportan aproximadamente 500 casos por año con una mortalidad hasta del $32 \%$ en su presentación severa (7).

Por el compromiso multiorgánico, la infección por S. typhi tiene diversas manifestaciones clínicas, de ahí su reconocimiento como "la gran simuladora". Las manifestaciones más frecuentes son gastrointestinales, sin embargo, las extraintestinales, que resultan como complicaciones secundarias de la infección por Salmonella, se producen entre el 10\%-15\% $(4,9)$, y pueden ser sangrado, perforación gastrointestinal, encefalopatía tifoidea (3\%-5\%), hepatitis con o sin colestasis (26\%) que puede tener un curso fulminante (menos del 5\%) asociado frecuentemente con coinfección por hepatitis $\mathrm{A} \mathrm{y} \mathrm{E}$ (10-12) y compromiso hematológico $(7,10)$.

\section{Fisiopatología}

La Salmonella typhi es un bacilo Gram negativo que hace parte de la familia de las enterobacterias y puede ser enté- rica o Bongori (13). Es una bacteria anaerobia facultativa, no formadora de esporas $(5,13)$. Dentro de su estructura tiene antígenos lipopolisacáridos $\mathrm{O} 9$ y O12, antígeno de la proteína flagelar $\mathrm{Hd}$, y antígeno polisacárido capsular Vi, que le confieren su capacidad virológica y que se han relacionado con la severidad del compromiso hepático $(2,4)$.

El compromiso extraintestinal por Salmonella depende del tamaño del inóculo, la virulencia del serotipo, la respuesta inmune del huésped y la presencia de factores locales protectores (7). El tamaño del inóculo determina la duración del período de incubación y el inicio de la bacteriemia que es, finalmente, la causa de las infecciones extraintestinales. Sin embargo, no se conoce la cantidad de inóculo necesaria para causar un compromiso extraintestinal específico $(2,7,14)$. La virulencia depende de la capacidad del microorganismo (MO) para invadir las células reticuloendoteliales de órganos a distancia, la liberación de endotoxinas, y la producción de invasina, una proteína necesaria para el reconocimiento de la bacteria por células no fagocíticas $(7,15)$.

Una vez ingerido el $\mathrm{MO}$, se activan 2 mecanismos que le permiten sobrevivir al ácido gástrico $(7,16)$ : el primero corresponde a un sistema inducible de homeostasis de $\mathrm{pH}$ que se activa con $\mathrm{pH}$ por debajo de 4,0; el segundo involucra la síntesis de proteínas de choque ácido que le permiten sobrevivir a un $\mathrm{pH}$ de $3,3(7,16)$. Cuando llega al intestino delgado, la bacteria es internalizada por las células $\mathrm{M}$ de las placas de Peyer, luego es transportada al tejido linfoide intestinal, desde donde se disemina en forma hematógena (4). Esta diseminación permite la migración bacteriana, siendo reconocida por el sistema reticuloendotelial de órganos distantes, en donde es capaz de sobrevivir y multiplicarse por un período de 7-14 días, después del cual se produce la fase de bacteriemia con el compromiso secundario de órganos como hígado, bazo, medula ósea y vesícula biliar $(2,4)$. Este último sitio es el reservorio de portadores asintomáticos hasta en el 5\% de los pacientes ( 7 , 17), situación que se facilita por la producción de cálculos biliares que permiten la fijación del microorganismo con formación de biopelícula, una cubierta de exopolisacáridos cuya función es proteger las estructuras bacterianas de la acción de antimicrobianos circulantes (17) (figura 1). No se conocen con claridad los mecanismos involucrados en la lesión hepática, pero se sabe que puede originarse por invasión directa del microorganismo o por daño inmunológico asociado a la endotoxemia $(2,4,14)$. La respuesta inmune del huésped también es un factor determinante en la patogénesis y la severidad de la disfunción orgánica secundaria de infección por Salmonella $(4,7,15)$. La consecuente liberación de citocinas proinflamatorias por los macrófagos es la responsable de la fase sintomática de la fiebre tifoidea $(4,7,15)$. 


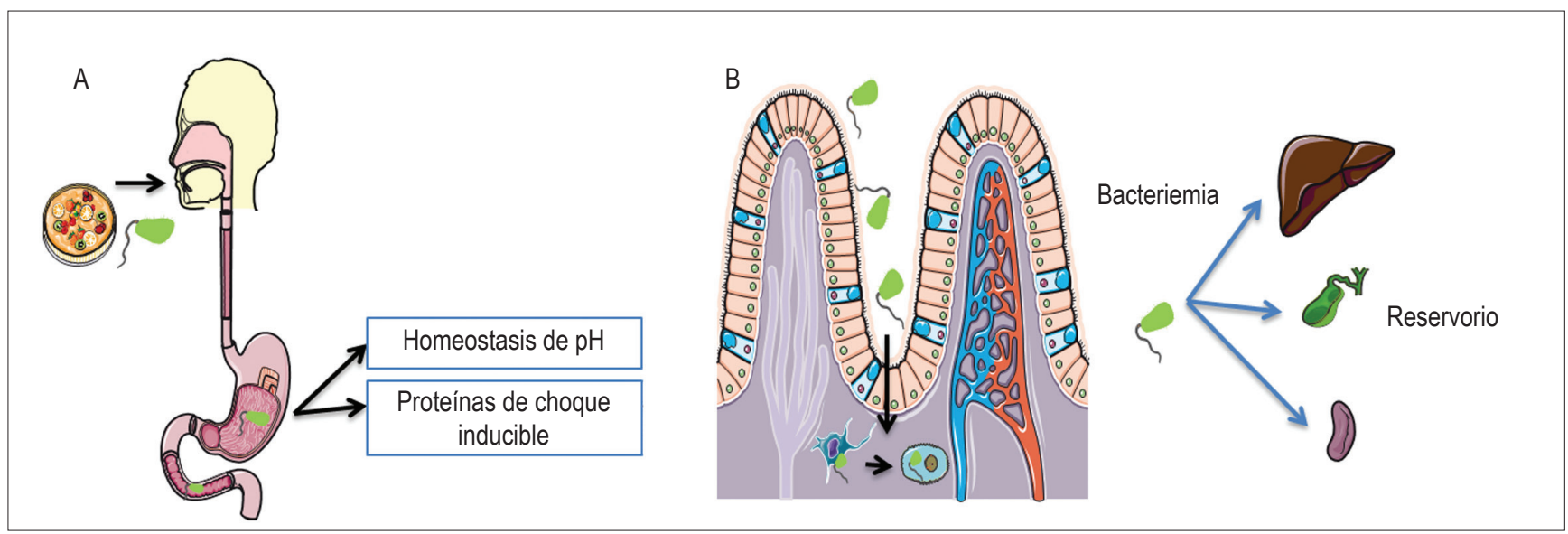

Figura 1. Fisiopatología de la infección por Salmonella. A. Activación de 2 mecanismos para resistencia al ácido gástrico. B. Internalización bacteriana por células M intestinales, migración extraintestinal por vía hematógena a órganos principales, hígado, vesícula biliar (reservorio) y bazo.

\section{Manifestaciones clínicas}

Las manifestaciones clínicas de la hepatitis por Salmonella son similares a las que se encuentran en la fiebre entérica, sin embargo, la ictericia puede ser un marcador importante en el diagnóstico del compromiso hepático secundario $(2,3,7)$. Se encuentra aproximadamente en el $12,2 \%$ de los pacientes ( 3 ), en general se produce al inicio de los síntomas, durante la primera semana del cuadro febril, y se resuelve con el cuadro clínico, a diferencia de la hepatitis viral, en la cual la fiebre desaparece cuando sobreviene la ictericia, o en otras palabras, con la ictericia desaparecen los síntomas prodrómicos $(2,13,18,19)$. La hepatomegalia se encuentra en todos los pacientes con hepatitis por Salmonella (18-20), así como pueden cursar con esplenomegalia; estas visceromegalias pueden ser evidentes en el examen físico o por ecografía abdominal (20). Además de las manifestaciones sistémicas que pueden apoyar el diagnóstico como miocarditis, insuficiencia renal, y alteraciones hemorrágicas y cutáneas (como la presencia de exantema $[30 \%])(7,9,15,17)$, también es frecuente encontrar bradicardia, a pesar de la fiebre, y esta situación es una característica clásica de la entidad $(1,2,4$, 7). Las manifestaciones neuropsiquiátricas se presentan en $10 \%-40 \%$ de los casos y son complicaciones importantes y discapacitantes, dado que se asocian con severidad en la presentación clínica; estas incluyen delirio, estupor, coma, meningitis por tifoidea, encefalomielitis, síndrome de Guillan-Barré, psicosis, manía o apatía. Estas manifestaciones pueden confundirse con encefalopatía hepática, sin embargo, la presencia de asterixis permite hacer el diagnóstico diferencial $(2,4,7)$.

\section{DIAGNÓSTICO}

\section{Alteraciones bioquímicas}

Todos los pacientes con hepatitis por Salmonella muestran alteraciones en el perfil hepático (1-3). La elevación de transaminasas se encuentra hasta en el 94\% de los casos alcanzando valores entre 3 a 5 veces el límite superior (2). A diferencia de la hepatitis viral, en la que se encuentran valores superiores a 1000 UI, en la hepatitis por Salmonella los valores no superan las 300 UI (2). Otros hallazgos frecuentes son hiperbilirrubinemia, generalmente entre 2 y $6 \mathrm{mg} / \mathrm{dL}$, aunque puede llegar hasta 10 a $16 \mathrm{mg} / \mathrm{dL}$ con predominio de la bilirrubina directa $(1,2,7)$; la fosfatasa alcalina puede ser normal o discretamente elevada, aunque se han descrito casos de hepatitis colestásica (10); la trombocitopenia se presenta hasta en el $25 \%$ de los pacientes $(1,2,7)$; la relación alaninoaminotransferasa/lactato deshidrogenasa (ALT/LDH) menor de 4 puede ser un indicador útil para diferenciar la hepatitis por Salmonella de la hepatitis viral, en donde la relación es mayor de $5(1,2)$. En las hepatitis virales, se hace evidente un pico de ALT, mayor elevación de bilirrubinas con discreta elevación en la $\mathrm{LDH}$, a diferencia de la hepatitis por Salmonella, en la que predomina la elevación de LDH con una menor elevación de transaminasas y bilirrubinas $(1,2)$. En el hemograma se puede encontrar 
leucopenia, anemia y trombocitopenia, que son hallazgos menos frecuentes en hepatitis viral (9).

\section{Métodos diagnósticos}

Los síntomas que se presentan en la hepatitis por Salmonella son inespecíficos, por lo tanto, el cuadro clínico puede ser indistinguible de otras causas de ictericia y fiebre, siendo las entidades más frecuentes las mostradas en la tabla 1 (2, $11,21-25)$.

Tabla 1. Diagnósticos diferenciales en pacientes con fiebre, ictericia y hepatitis.

\begin{tabular}{l}
\hline Infecciones \\
\hline Malaria, leptospira, fiebre amarilla, dengue \\
Brucelosis, ricketssia, brucelosis, tuberculosis \\
Citomegalovirus, virus Epstein-Barr \\
\hline Neoplasias \\
\hline Linfoma de Hodgkin \\
\hline Inflamatorias \\
\hline Tromboflebitis séptica \\
\hline
\end{tabular}

Para el diagnóstico, se requiere del aislamiento de la Salmonella en sangre, medula ósea, heces, secreción del tubo digestivo o lesiones cutáneas $(2,4,17)$. El hemocultivo es el método diagnóstico más usado, se encuentra positivo solo en el $60 \%$ a $80 \%$ de los pacientes, y su sensibilidad es mayor en la primera semana de la presentación clínica $(2,4)$. El cultivo en medula ósea es la prueba de oro para el diagnóstico, ya que es más sensible que el hemocultivo, con positividad en el $95 \%$ de los casos $(2,4)$. La utilidad del coprocultivo depende de la cantidad de materia fecal cultivada, y es positivo solo en el $30 \%$ de los pacientes, por lo que no es un examen de rutina $(2,4)$. La prueba de Widal, descrita en el siglo XIX, es una técnica de aglutinación de anticuerpos contra el lipopolisacárido $\mathrm{O}$ y el antígeno $\mathrm{H}$ flagelar por dilución seriada $(17,21)$; es una prueba serológica poco utilizada en la actualidad, ya que presenta reacción cruzada con muchos otros antígenos y requiere que las muestras se tomen en el período activo o en la convalecencia, por lo cual se considera que es una técnica metodológicamente difícil $(17,21)$.

\section{Hallazgos histopatológicos}

La biopsia hepática es un método que puede ser útil para establecer diagnósticos diferenciales, sin embargo, no se realiza de forma rutinaria $(2-4,16,19,26)$. En histología, la hepatitis por Salmonella se caracteriza por la presencia de nódulos con células de Kupffer hiperplásicas, cambios por infiltración focal de células mononucleares e infiltración grasa $(2-4,16,19,26)$.

\section{TRATAMIENTO}

Más del 90\% de los pacientes con fiebre tifoidea podrán ser manejados de forma ambulatoria con antibióticos orales, sin embargo, un pequeño porcentaje requerirá de manejo intrahospitalario (4). Estos pacientes, además del adecuado cubrimiento antibiótico, requerirán de cuidados por enfermería, nutrición adecuada y control de líquidos y electrolitos $(4,27)$; esta vigilancia estrecha permitirá el reconocimiento temprano de las complicaciones asociadas con esta entidad $(1,27)$. La ampicilina, cloranfenicol y trimetroprima-sulfametoxazol (TMP-SMX) fueron por muchos años los antibióticos de primera línea en el tratamiento de la fiebre entérica, pero su uso empírico e indiscriminado facilitó la generación de cepas resistentes $(21$, 28). En 1972 se aislaron cepas resistentes al cloranfenicol en países como México, India, Vietnam y Corea (29); en 1980 se aislaron cepas resistentes a los 3 medicamentos de primera línea, dando origen a las cepas conocidas como resistentes a múltiples medicamentos (29).

Ante la necesidad de controlar la epidemia de dichas cepas se comenzaron a utilizar fluoroquinolonas, específicamente ciprofloxacina $(5,29)$, y por su efectividad en cepas resistentes, además de su adecuada absorción oral, buena tolerancia, mínimos efectos secundarios y bajo costo, se introdujo como el tratamiento de elección en esta entidad $(27,30,31)$. Las fluoroquinolonas son antibióticos sintéticos que actúan impidiendo la acción de la $\mathrm{ADN}$ girasa y la topoisomerasa, proteínas necesarias para la replicación y duplicación del ADN (32). Tienen una adecuada penetración tisular, y actúan sobre las formas inmóviles intracelulares de S. typhi, logrando, además, adecuados niveles en la vesícula biliar, a diferencia de otros antimicrobianos (27); también producen una rápida respuesta terapéutica con aclaramiento de la fiebre en 3 a 5 días, con una tasa de recuperación mayor al $96 \%$ en pacientes infectados por cepas sensibles $(4,28)$. Hasta el momento, no se ha demostrado diferencia en la eficacia entre de quinolonas de segunda y tercera generación (28). En un estudio realizado por Koirala y colaboradores, comparando ofloxacina con gatifloxacina en el tratamiento de cepas resistentes a múltiples medicamentos, no se demostró superioridad en la prevención de falla terapéutica (33). No obstante, el uso de quinolonas de tercera y cuarta generación se ha relacionado con resistencia cruzada con otras quinolonas que se requieren para el tratamiento de diversas patologías como tuberculosis (5). Por lo tanto, la elección del antibiótico debe realizarse con base en la resistencia antimicrobiana 
local $(19,29,30)$. Las quinolonas parenterales son el antibiótico de elección en el manejo de la fiebre tifoidea con complicaciones y deben ser administradas mínimo por 10 días $(4,27)$ (tabla 2). Se debe descartar meningitis por Salmonella en pacientes con manifestaciones neurológicas, delirio, estupor y cambios agudos del comportamiento (2, 4). En esos pacientes, la adición de esteroides disminuye la mortalidad $(1,4,18)$.

Tabla 2. Tratamiento para fiebre tifoidea severa.

\begin{tabular}{|c|c|c|}
\hline & Primera línea & Segunda línea \\
\hline $\begin{array}{l}\text { Sensible a } \\
\text { quinolonas }\end{array}$ & $\begin{array}{l}\text { Ciprofloxacina/ } \\
\text { ofloxacina } \\
15 \mathrm{mg} / \mathrm{kg} \text { por } 10-14 \\
\text { días }\end{array}$ & $\begin{array}{l}\text { Cloranfenicol* }(100 \mathrm{mg} / \mathrm{kg} \text { por } \\
14-21 \text { días) } \\
\text { Ampicilina* }(100 \mathrm{mg} / \mathrm{kg} \text { por } \\
14 \text { días) } \\
\text { TMP-SMX* }(8-40 \mathrm{mg} / \mathrm{kg} \text { por } 14 \text { días) }\end{array}$ \\
\hline $\begin{array}{l}\text { Resistente a } \\
\text { quinolonas }\end{array}$ & $\begin{array}{l}\text { Ceftriaxona o } \\
\text { Cefotaxima (60-80 } \\
\text { mg/kg por 10-14 días) } \\
\text { Azitromicina (8-10 } \\
\text { mg/kg por } 7 \text { días) }\end{array}$ & $\begin{array}{l}\text { Cefixima (20 mg/kg por 7-14 } \\
\text { días) }\end{array}$ \\
\hline
\end{tabular}

* Antibióticos de primera línea útiles en regiones con baja tasa de resistencia.

TMP-SMX: trimetropima y sulfametoxazol.

Otros esquemas de tratamiento incluyen cefalosporinas de tercera generación (ceftriaxona, cefixima, cefotaxima, cefoperazona) y azitromicina, ampliamente utilizada en población pediátrica, con una tasa de curación del $95 \%$ $(5,34)$. Antibióticos como aztreonam, imipenem y meropenem son esquemas de tercera línea que se reservan para cepas con susceptibilidad disminuida a quinolonas, definidas por la resistencia al ácido nalidíxico (28).

\section{CONCLUSIÓN}

La fiebre tifoidea es una entidad patológica de alto impacto en países en vías de desarrollo. Es producida por Salmonella entérica, serotipo typhi, con capacidad para invadir la mucosa intestinal y órganos a distancia por diseminación hematógena. Las manifestaciones clínicas más frecuentes son gastrointestinales, sin embargo puede presentar complicaciones extraintestinales que, a pesar de ser poco frecuentes, pueden resultar altamente mortales. La hepatitis por Salmonella es una complicación poco frecuente, cuyas manifestaciones clínicas y bioquímicas pueden ser indistinguibles de otras causas de hepatitis, frecuentes en países tropicales.

Dentro de los hallazgos clínicos más importantes se encuentran la ictericia que coincide con el cuadro febril, a diferencia de la hepatitis viral. Todos los pacientes con hepatitis por Salmonella tienen alterado el perfil hepático. En general, las transaminasas alcanzan valores no mayores a 300 UI, y en algunos casos se puede encontrar también hiperbilirrubinemia con predominio de la bilirrubina directa y trombocitopenia.

Para el diagnóstico se deben considerar los hallazgos clínicos y el aislamiento del microorganismo por cultivos, ya sea por hemocultivo o cultivo de medula ósea.

El tratamiento de elección en estos pacientes son las quinolonas vía parenteral por 10 a 14 días; aun así, se deben considerar las tasas de resistencia local para la elección del antibiótico. A pesar de ser una entidad de buen un pronóstico, reviste serias dificultades para su diagnóstico, de allí la importancia de ser reconocida de forma temprana como una complicación de la fiebre tifoidea en pacientes con fiebre, ictericia y hepatitis.

\section{REFERENCIAS}

1. Karoli R, Fatima J, Chandra A, Singh G. K hepatitis: An uncommon complication of a common disease. J Fam Med Prim care. 2012;1(2):160-2.

2. Pramoolsinsap C, Viranuvatti V. Salmonella hepatitis. J Gastroenterol Hepatol. 1998; 13:745-50.

3. Ahmed A, Ahmed B. Jaundice in typhoid patients: Differentiation from other common causes of fever and jaundice in the tropics. Ann Afr Med. 2010;9(3):135-40.

4. Parry CM, Hien TT, Dougan G, White NJ, Farrar JJ. Typhoid fever. N Engl J Med. 2002;347(22):1770-82.

5. Wain J, Hendrikse R, Mikoleit ML, Keddy KH, Ochiai R. Typhoid fever. Lancet. Elsevier Ltd. 1981;79(panel 1):791-3.

6. Otero W. Hepatitis con ictericia y fiebre. Syllabus 1er Curso Internacional de Medicina Tropical y Enfermedades olvidadas. Universidad Nacional de Colombia e Instituto Suizo de Medicina Tropical. 2007

7. Huang DB, DuPont HL. Problem pathogens: Extraintestinal complications of Salmonella enterica serotype Typhi infection. Lancet Infect Dis. 2005;5:341-8.

8. Instituto Nacional de Salud. Comportamiento epidemiológico de la vigilancia de fiebre tifoidea y paratifoidea en Colombia, 2011. Inf Quinc Epidemiológico Nac. 2013;18(16):164-74.

9. Parry CM, Thompson C, Vinh H, Chinh NT, Phuong LT, Ho VA, et al. Risk factors for the development of severe typhoid fever in Vietnam. BMC Infect Dis. 2014;14(1):73.

10. Albayrak A, Gunbey SS, Aktas F. Cholestatic hepatitis due to Salmonella typhi. Clin Pract. 2011;1(313):22-3.

11. Husain EH. Fulminant hepatitis in typhoid fever. J Infect Public Health [Internet]. King Saud Bin Abdulaziz University for Health Sciences. 2011;4(3):154-6.

12. Ratnayake EC, Shivanthan C, Wijesiriwardena BC. Cholestatic hepatitis in a patient with typhoid fever - A case report. Acta Gastroenterol Belg. BioMed Central Ltd. 2012;75(1):65. 
13. Longo D, Kasper D, Jameson JL, Fauci AS, Hauser SL, Loscalzo J. Harrison Principios de Medicina Interna. 18.a edición. 2012.

14. Dougan G, Baker S. Salmonella enterica Serovar Typhi and the pathogenesis of typhoid fever. Annu Rev Microbiol. 2014;68:317-36.

15. De Jong HK, Parry CM, Van der Poll T, Wiersinga WJ. Host-pathogen interaction in invasive salmonellosis. PLoS Pathog. 2012;8(10):1-9.

16. Waddington CS, Darton TC, Woodward WE, Angus B, Levine MM, Pollard AJ. Advancing the management and control of typhoid fever: A review of the historical role of human challenge studies. J Infect.Elsevier Ltd. 2014;68(5):405-18.

17. Waddington CS, Darton TC, Pollard AJ. The challenge of enteric fever. J Infect. Elsevier Ltd. 2014;68:S38-50.

18. Rungta N, Singhi S, Chaudhary D, Varghese G, Bhalla A, Karthi N, et al. Tropical fevers: Management guidelines. Indian J Crit Care Med. 2014;18:62.

19. Khosla SN. Typhoid hepatitis. Postgraduated Med J. 1990;66:923-5.

20. Nadhim Younis S. The role of abdominal ultrasound in the diagnosis of typhoid fever: An observational study. Travel Med Infect Dis. Elsevier Ltd. 2013;1-4.

21. Darton TC, Blohmke CJ, Pollard AJ. Typhoid epidemiology, diagnostics and the human challenge model. Curr Opin Gastroenterol. 2014;30:7-17.

22. Bathia S, Prabhu M, Saravu M, Docherla B. Spectrum of hepatic dysfunction in enteric fever. Int $\mathrm{J}$ Infect Dis. 2010;14:e214-5.

23. Isa SE, Onyedibe KI, Okolo MO, Abiba AE, Mafuka JS, Simji GS, et al. A 21-year-old student with fever and profound jaundice. PLoS Negl Trop Dis. 2014;8(1):3.

24. Schindler M, Kreplak N, Acevedo L, Damis H, Marquez G, Ardaiz M. Ictericia febril colestásica como forma de presentacion de linfoma de Hodgkin. Med Buenos Aires. 2014;74:60-1.

25. Zimhony O, Katz M. A patient with fever and jaundice. QJM. 2012;105:381-2.

26. Madrid M, Huguet J. Enfermedades hepáticas infecciosas. GH Contin. 2006; 5:218-21.

27. WHO. Background document: The diagnosis, treatment and prevention of typhoid fever. Commun Dis Surveill Response Vaccines Biol. 2003.

28. Tatavarthy A, Luna V, Amuso PT. How multidrug resistance in typhoid fever affects treatment options. Ann N Y Acad Sci. 2014; 1323:76-90.

29. Zaki S, Karande S. Multidrug-resistant typhoid fever: A review. J Infect Dev Ctries. 2011;5(5):324-7.

30. Thaver D, Zaidi AKM, Critchley J, Azmatullah A, Madni SA, Bhutta Z. Fluoroquinolones for treating typhoid and paratyphoid fever (enteric fever). Cochrane Database Syst Rev. 2008; (10).

31. Thaver D, Zaidi AKM, Critchley J, Azmatullah A, Madni SA, Bhutta Z. A comparison of fluoroquinolones versus other antibiotics for treating enteric fever: meta-analysis. BMJ. 2009;338:b1865.

32. Drlica K, Zhao X. DNA gyrase, topoisomerase IV, and the 4-quinolones. Microbiol Mol Biol Rev. 1997;61:377.

33. Koirala S, Basnyat B, Arjyal A, Shilpakar O, Shrestha K, Shrestha R, et al. Gatifloxacin versus ofloxacin for the treatment of uncomplicated enteric fever in nepal: an openlabel, randomized, controlled trial. PLoS Negl Trop Dis. 2013;7(10).

34. Trivedi N, Shah P. A meta-analysis comparing the safety and efficacy of azithromycin over the alternate drugs used for treatment of uncomplicated enteric fever. J Postgr Med. 2012;58(2):112-8. 